\title{
Fast ignition of inertial fusion targets by laser-driven carbon beams
}

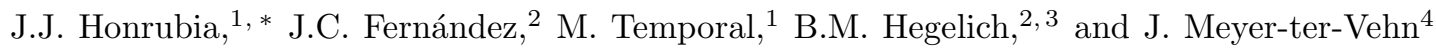 \\ ${ }^{1}$ ETSI Aeronáuticos, Universidad Politécnica de Madrid, Madrid-28040, Spain \\ ${ }^{2}$ Los Alamos National Laboratory, Los Alamos, NM-87544, USA \\ ${ }^{3}$ Fakultät für Physik, LMU München, D-85748 Garching, Germany \\ ${ }^{4}$ Max-Planck-Institut für Quantenoptik, D-85748 Garching, Germany
}

(Dated: September 28, 2018)

\begin{abstract}
Two-dimensional simulations of ion beam driven fast ignition are presented. Ignition energies of protons with Maxwellian spectrum and carbon ions with quasimonoenergetic and Maxwellian energy distributions are evaluated. The effect of the coronal plasma surrounding the compressed deuteriumtritium is studied for three different fuel density distributions. It is found that quasimonoenergetic ions have better coupling with the compressed deuterium-tritium and substantially lower ignition energies. Comparison of quasimonoenergetic carbon ions and relativistic electrons as ignitor beams shows similar laser energy requirements, provided that a laser to quasimonoenergetic carbon ion conversion efficiency around $10 \%$ can be achieved.
\end{abstract}

PACS numbers: $52.38 . \mathrm{Kd}, 52.65 . \mathrm{Ww}, 52.57 . \mathrm{Kk}$

\section{INTRODUCTION}

Fast ignition by laser-driven ion beams has been proposed [1, 2] as an alternative to the standard scheme of fast ignition by relativistic electrons [3]. It offers the advantages of the classical interaction of ion beams with compressed fuels, their much more localized energy deposition, and the stiffer ion transport with the possibility of beam focusing. Proton fast ignition (PFI) is a promising option because of the high laser-to-proton conversion efficiencies $(\approx 6-12 \%)$ found in experiments for Maxwellian energy distributions [4, 5]. Novel target geometries such as flat-top cone targets seem to be appropriate to increase those efficiencies [6]. Monoenergetic protons may have a better coupling with the compressed core, but the relatively low conversion efficiencies $(\approx 1 \%[7])$ found so far hamper their application to fast ignition.

Studies on PFI have shown that ignition energies can be reduced substantially by using a sequence of two beams [8]. Numerical simulations show that fuel targets compressed to $\rho=500 \mathrm{~g} / \mathrm{cm}^{3}$ can be ignited by two Maxwellian proton beams with temperature $\mathrm{T}_{p}=4 \mathrm{MeV}$ and a total energy of $8 \mathrm{~kJ}$ [9]. Assuming a conversion efficiency of $10 \%$, the required laser energy is about $80 \mathrm{~kJ}$, that is of the same order than the energies envisioned for future fast ignition facilities such as HiPER (High Power laser Energy Research) [10]. However, the PFI scheme requires to place the proton source near the compressed fuel inside a re-entrant cone to shield the source from the plasma coming from the imploding shell. Thus, the capsule implosion and fuel compression will be relatively complex due to the flow perturbations induced by the cone. A complete simulation of an indirectly driven target ignited by laser-driven protons can be found in [11].

Recently, particle-in-cell (PIC) simulations of the in-

*Electronic address: javier.honrubia@upm.es teraction of short pulses of circularly polarized laser light with thin foils at intensities of $10^{22} \mathrm{~W} / \mathrm{cm}^{2}$ have shown that it is possible to accelerate ions up to hundreds of $\mathrm{MeV}$ with quasimonoenergetic energy distributions and small divergence angles [12, 13, 14, 15, 16, 17, 18]. In this scheme, that we refer to as RPA (Radiation Pressure Acceleration), the whole foil is accelerated by radiation pressure with laser-to-ion conversion efficiencies around $10 \%$ [14]. The so-called laser break-out afterburner (BOA) scheme has been also proposed to generate almost perfectly collimated quasimonoenergetic ions with conversion efficiencies of a few percent 19] at laser intensities about $10^{21} \mathrm{~W} / \mathrm{cm}^{2}$. These new schemes are suitable to accelerate ions heavier than protons to the energies required for fast ignition applications. Fernández et al. [20, 21] suggested the use of quasimonoenergetic carbon ions with kinetic energies of a few hundreds of $\mathrm{MeV}$ to ignite pre-compressed fusion targets [22]. Use of heavier ions has been studied in Ref [23]. One advantage of quasimonoenergetic ion beams is the possibility to place the source far from the compressed core avoiding the use of a re-entrant cone. However, the feasibility of the carbon ion fast ignition scheme (CFI) relies on the demonstration of conversion efficiencies comparable to those found for protons [20, 24]. Here, we assume a laser to quasimonoenergetic carbon ions conversion efficiencies of about $10 \%$ and compare the potential of the PFI and CFI schemes for fast ignition.

The paper is organized as follows. The simulation model is briefly summarized in Sec. II. Energy deposition and ignition energies of proton and carbon ions with different deuterium-tritium (DT) density distributions are studied in Sec. III. Ignition energies of ions and relativistic electrons are compared in Sec. IV. Finally, conclusions and future developments are summarized in Sec. V. 

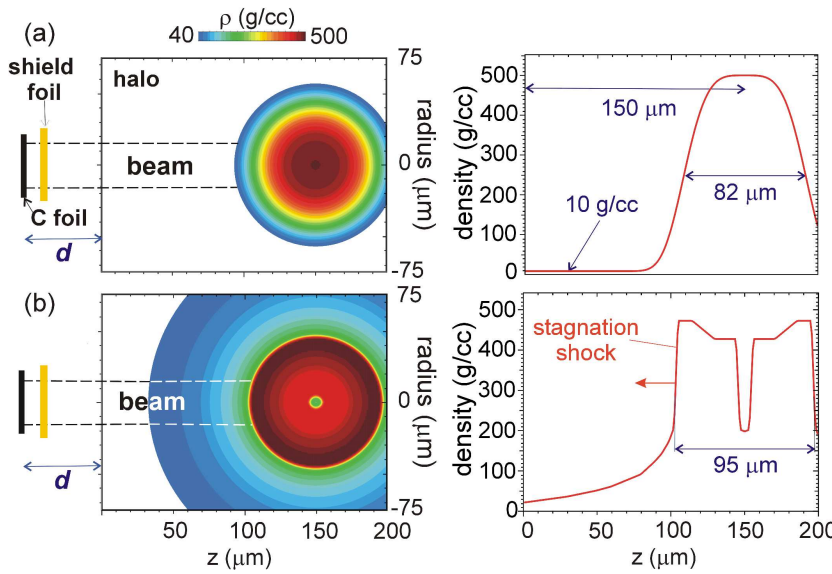

(b)

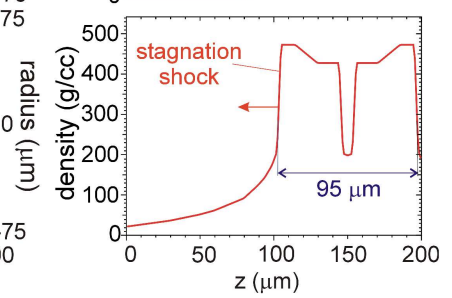

FIG. 1: Density maps and radial profiles of the precompressed targets considered. (a) Super-Gaussian density distribution of the spherical blob, and (b) density distribution taken from Fig. 8 of Ref. [29]. $d$ is the distance from the ion source to the simulation box.

\section{SIMULATION MODEL}

We assume perfectly collimated cylindrical ion beams of $30 \mu \mathrm{m}$ diameter impinging on a DT blob with a peak density of $500 \mathrm{~g} / \mathrm{cm}^{3}$. Recent implosion calculations have shown that it is possible to achieve such high densities in capsules of $0.59 \mathrm{mg}$ of DT with laser energies around $200 \mathrm{~kJ}$ [25, 26]. It is worth recalling that densities higher than $500 \mathrm{~g} / \mathrm{cm}^{3}$ were obtained in direct-drive implosions conducted by $10 \mathrm{~kJ}$ laser pulses [27]. We consider the two different configurations of the compressed DT shown in Fig. 1. The first one is a simplification of the configuration obtained from the two-dimensional (2D) implosion calculations of cone-targets presented elsewhere [25, 28]. The second one is similar to that obtained by Clark and Tabak [29] from one-dimensional (1D) implosion calculations to get an almost isochoric configuration of the compressed fuel. As shown in Ref. [29], this profile corresponds to the time of maximum density ( $\mathrm{t}=35.9 \mathrm{~ns}$ in Fig. 8 of [29]) of a target with $0.9 \mathrm{mg}$ of DT compressed by a laser pulse of $480 \mathrm{~kJ}$. The main differences between the two density distributions shown in Figs. 1(a) and (b) are the higher $\rho \mathrm{R}$ of the plasma surrounding the dense core and the steeper density ramp of the Clark \& Tabak's target. Since the DT plasma is stagnated at the time of peak $\rho R$, we assume that the DT is initially at rest with an uniform temperature of $300 \mathrm{eV}$, with exception of the central dip of the target shown in Fig. 1(b), that has an initial temperature around $1 \mathrm{keV}$ to get pressure balance between the hot spark and the dense fuel.

Calculations have been performed with the 2-D radiation-hydrodynamics code SARA, that includes fluxlimited electron conduction, multigroup radiation transport, ion energy deposition, DT fusion reactions and $\alpha$ particle transport [30, 31]. We validated our simulation model by comparing the ignition energy $(\approx 9 \mathrm{~kJ})$ of mo-

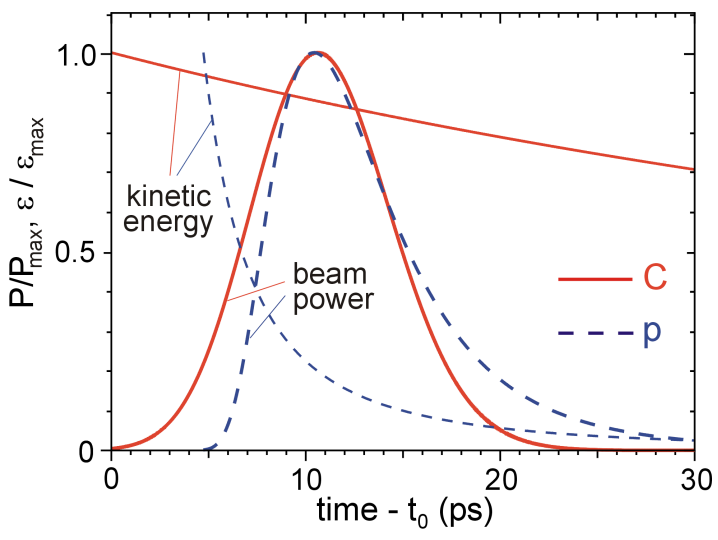

FIG. 2: Beam power and ion kinetic energy at the left surface of the simulation box as a function of time. The dashed lines correspond to a Maxwellian proton beam with $T_{p}=4 \mathrm{MeV}$, $d=0.5 \mathrm{~mm}, \mathrm{t}_{0}=0, \epsilon_{\max }=58 \mathrm{MeV}$ and $\mathrm{P}_{\max }=1.12 \mathrm{PW}$. The solid lines correspond to a Gaussian carbon ion beam with $\delta \epsilon / \epsilon_{0}=0.1, d=1.35 \mathrm{~cm}, \mathrm{t}_{0}=158 \mathrm{ps}, \epsilon_{\max }=458 \mathrm{MeV}$ and $\mathrm{P}_{\max }=1.12 \mathrm{PW}$.

noenergetic proton beams impinging on an uniform DT plasma precompressed to $400 \mathrm{~g} / \mathrm{cm}^{3}$ with that obtained by Atzeni et al. 32$](\approx 8.5 \mathrm{~kJ})$.

\section{A. Ion pulse on target}

The simulation box is shown in Fig. 1 Ions come from the left and propagate towards the blob through a low density plasma. We assume that ions are generated instantaneously with a Maxwellian energy distribution for protons and a Gaussian energy distribution with a given energy spread for carbon ions. If the energy spread is $10 \%$ (full width at half-maximum, FWHM), we refer to this last distribution as "quasimonoenergetic" throughout the paper. Instantaneous emission of the beam ions is assumed because the spread of the time of flight $(\approx 10$ ps) from the source to the target is much longer than the typical ion acceleration time $(\approx 1 \mathrm{ps})$.

We used analytical formulas [8, 33] to compute the kinetic energy and the beam power on target of ions accelerated instantaneously at a distance $d$. Thus, ions with a Gaussian energy distribution $N(\epsilon)=$ $N_{0} \sqrt{\alpha} \exp \left\{-\alpha\left[\left(\epsilon-\epsilon_{0}\right) / \Delta\right]^{2}\right\} / \Delta \sqrt{\pi}$ have a power on target

$$
P(t)=\frac{N_{0} \sqrt{\alpha} d^{4} m_{i}^{2}}{2 \Delta \sqrt{\pi} t^{5}} \exp \left\{-\alpha\left[d^{2} m_{i} / 2 \Delta t^{2}-\epsilon_{0} / \Delta\right]^{2}\right\},
$$

where $N_{0}$ is the total number of ions $\left(E / \epsilon_{0}\right)$, E the beam energy, $\epsilon_{0}$ the mean ion kinetic energy, $\Delta$ the energy spread (FWHM), $m_{i}$ the ion rest mass and $\alpha=$ $4 \ln (2)$. Ion kinetic energy on target is given by $\epsilon(t)=$ $1 / 2 m_{i} c^{2}(d / c t)^{2}$. Ions with a Maxwellian energy distribution $N(\epsilon)=2 N_{0} \sqrt{\epsilon} \exp [-(\epsilon / k T)] / \sqrt{\pi}(k T)^{3 / 2}$ have a 


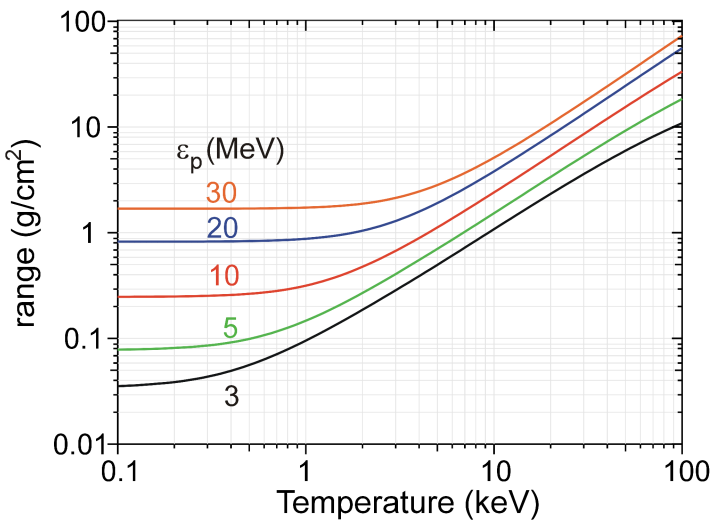

FIG. 3: Range of monoenergetic protons with a kinetic energy $\epsilon_{p}$ versus plasma temperature in DT at $400 \mathrm{~g} / \mathrm{cm}^{3}$.

power on target

$$
P(t)=\frac{8 E}{3 \sqrt{\pi} \tau}\left(\frac{\tau}{t}\right)^{6} \exp \left\{-(\tau / t)^{2}\right\},
$$

where $\mathrm{E}$ is the beam energy, $\tau=d /\left(2 k T / m_{i}\right)^{1 / 2}$ the pulse time scale and $T$ the ion temperature.

Beam power and ion kinetic energy of $10 \mathrm{~kJ}$ Maxwellian proton beam generated at $d=0.5 \mathrm{~mm}$ and quasimonoenergetic carbon ion beams generated at $d=$ $1.35 \mathrm{~cm}$ are shown in Fig. 2. These two distances have been chosen in order to have the same peak power and approximately the same pulse duration on target $(\approx 8$ ps) with both beams. It is worth noting that the almost negligible time spread of ions with quasimonoenergetic energy distributions allows to place the source at much higher distances $d$ than Maxwellian ions. Because beam focalization over distances of a few centimeters may be difficult, a number of focusing techniques have been proposed over the last years. Some of these techniques are: i) ballistic transport [34, 35], ii) focusing by fields self-generated in hollow microcylinders by intense subpicosecond laser pulses [36] and iii) focusing by magnetic lenses [37].

\section{B. Range lengthening}

Range of proton and carbon ions for different kinetic energies as a function of plasma temperature are shown in Figs. 3 and 4 , respectively. Calculations have been performed by pursuing the standard stopping power formalism [31]. Ion range increases when plasma electron velocities become comparable to the ion velocity. This effect is important for ions with Maxwellian energy distributions placed far from the fuel: indeed the decrease of ion kinetic energy with time (see Fig. 2) is balanced by their range lengthening as the DT is heated up, keeping the ion range almost constant with time [33]. On the contrary, range lengthening may be a disadvantage for quasimonoenergetic ions because their kinetic energy on

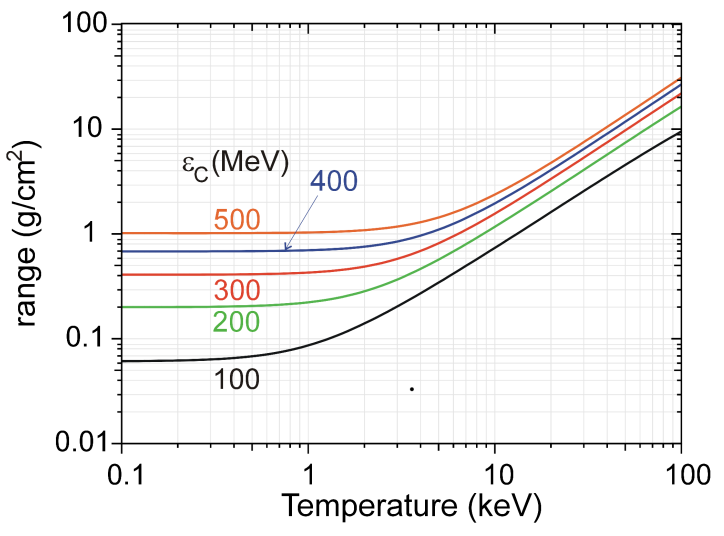

FIG. 4: Range of monoenergetic carbon ions with a kinetic energy $\epsilon_{C}$ versus plasma temperature in DT at $400 \mathrm{~g} / \mathrm{cm}^{3}$.

target has only a small variation with time when compared with Maxwellian ions (see Fig. (2)). However, range lengthening is less pronounced for ions with high kinetic energies per nucleon, as shown in Figs. 3 and 4 . For instance, the range of $400 \mathrm{MeV}$ carbon ions or $30 \mathrm{MeV}$ of protons increases only by a factor of $\approx 3$ for plasma temperatures from hundreds of $\mathrm{eV}$ to $10 \mathrm{keV}$ while the range of $5 \mathrm{MeV}$ protons increases by a factor of 20 for the same temperature interval. Nevertheless, unlike $400 \mathrm{MeV}$ carbon ions, the range of $30 \mathrm{MeV}$ protons at high plasma temperatures becomes much larger than the typical value of $\approx 1 \mathrm{~g} / \mathrm{cm}^{2}$ required for fast ignition, increasing considerably the ignition energy. In general, heavier ions carry more energy per unit of mass than protons for a given range, allowing target ignition with much lower ion number and beam currents.

\section{RESULTS}

\section{A. Energy deposition}

The energy deposited by proton and carbon ions with different energy distributions in different target configurations are compared in Fig. 5. Maxwellian ions are generated at a distance $d=0.5 \mathrm{~mm}$ from the target in order to limit their time spread. Left panels show the energy deposition in the target characterized by the super-Gaussian density distribution of Fig. 1(a). Most of the beam energy is deposited within the dense core in a volume determined by the ion spectrum and the range lengthening effect. As shown in Figs. 5(a-c), quasimonoenergetic beams have a more concentrated energy deposition than Maxwellian ions allowing for a coupling efficiency as high as $\eta_{c}=75 \%$ (defined as the fraction of the beam energy deposited at plasma densities $\rho \geq 200$ $\left.\mathrm{g} / \mathrm{cm}^{3}\right)$. Maxwellian ions have a more distributed energy deposition and lower coupling efficiencies, e.g. $62 \%$ for Maxwellian carbon ions and $65 \%$ for Maxwellian protons. The energy density maps shown in Figs. [5(a-c) have been 


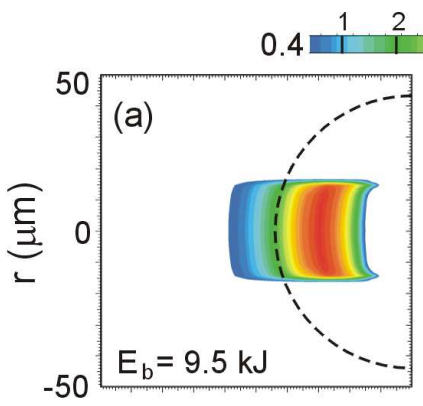

$\begin{array}{ll}3 & 4 \\ 1 & 1\end{array}$
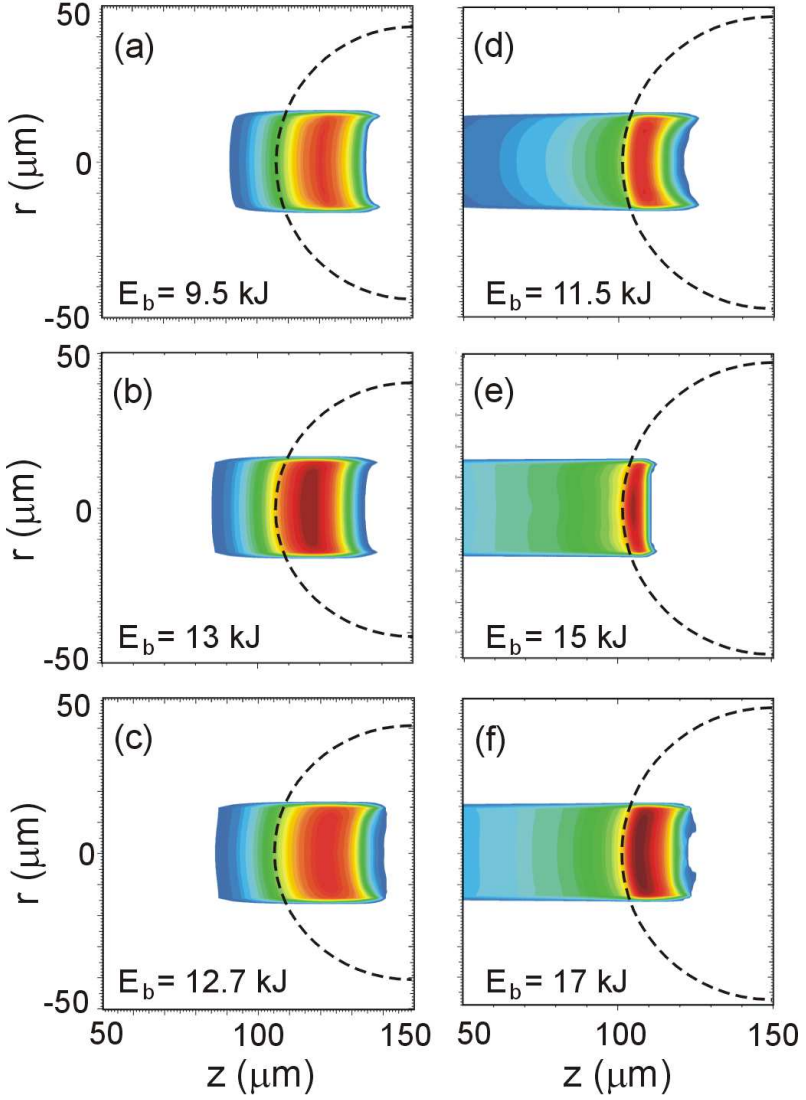

FIG. 5: Energy density in units of $10^{11} \mathrm{~J} / \mathrm{cm}^{3}$ deposited by ( $\mathrm{a}$ and $\mathrm{d}$ ) a quasimonoenergetic carbon ion beam with mean kinetic energy of $400 \mathrm{MeV}$ and $\delta \epsilon / \epsilon_{0}=0.1$ generated at a distance $d=1.35 \mathrm{~cm}$; (b and e) carbon beam with Maxwellian energy distribution and temperature $T_{C}=100$ $\mathrm{MeV}$ generated at $d=0.5 \mathrm{~mm}$; and (c and $\mathrm{f}$ ) proton beam with Maxwellian energy distribution and temperature $T_{p}=4$ $\mathrm{MeV}$ generated at $d=0.5 \mathrm{~mm}$. The left panels correspond to the target with the supergaussian density distribution and the right panels to the imploded target with the almost isochoric fuel configuration. Dashed lines show the initial position of the $\rho=200 \mathrm{~g} / \mathrm{cm}^{3}$ isocontour. The total ion beam energy $\mathrm{E}_{b}$ used in each simulation is shown.

obtained for beam energies $E_{b}$ equal to the minimum ignition energies $E_{i g}$. These last energies are $E_{i g}=9.5 \mathrm{~kJ}$ for quasimonoenergetic carbon ions, $13 \mathrm{~kJ}$ for Maxwellian carbon ions with a temperature $T_{C}=100 \mathrm{MeV}$ and 12.7 $\mathrm{kJ}$ for Maxwellian protons with $T_{p}=4 \mathrm{MeV}$. Here, temperatures $T_{C}$ and $T_{p}$ are the optimal beam temperatures for which the minimum ignition energies are obtained. It is worthwhile noting that despite the peak values of the energy densities shown in Figs. 5 (a-c) are around $4.6 \times 10^{11} \mathrm{~J} / \mathrm{cm}^{3}$, the ion beam energies necessary to obtain them are quite different due to the different coupling efficiencies found for each beam. It is also remarkable that Maxwellian protons and carbon ions have very similar ignition energies for the optimal beam temperatures,

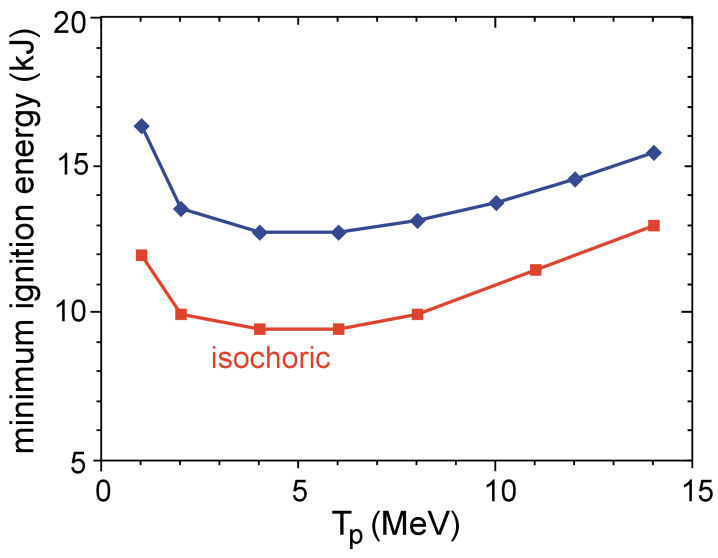

FIG. 6: Minimum ignition energies of the target shown in Fig. 1(a) heated by protons with a Maxwellian energy distribution of temperature $\mathrm{T}_{p}$. The ignition energies corresponding to an ideal isochoric fuel are also shown. The ion source - core distance is $d=0.5 \mathrm{~mm}$ in all cases.

while the energy for quasimonoenergetic carbon ions is about $25 \%$ lower. In general, quasimonoenergetic ions have a better coupling with the compressed core, allowing fuel ignition with beam energies substantially lower than those obtained for Maxwellian ions.

Right panels of Fig. [5 show the energy deposition for the more realistic, almost isochoric fuel configuration shown in Fig. 1(b). In this case, the higher areal density of the plasma surrounding the dense core reduces the beam coupling efficiencies to $\eta_{c}=40 \%, 23 \%$ and $39 \%$ for the cases (d), (e) and (f) of Fig. 5 , respectively. Thus, the ignition energies increase substantially, varying from $E_{i g}=11.5 \mathrm{~kJ}$ for quasimonoenergetic carbon ions to $17 \mathrm{~kJ}$ for Maxwellian protons. The corresponding energy densities are slightly higher in this case than for the super-Gaussian density distribution, ranging their peak values from $4.6 \times 10^{11} \mathrm{~J} / \mathrm{cm}^{3}$ for quasimonoenergetic carbon ions to $5.4 \times 10^{11} \mathrm{~J} / \mathrm{cm}^{3}$ for Maxwellian protons. The very low coupling efficiency found for Maxwellian carbon ions with a beam temperature $T_{C}=100 \mathrm{MeV}$ leads to an ignition energy higher than $20 \mathrm{~kJ}$. This energy can be reduced by raising the beam temperature to allow a deeper penetration of carbon ions in the dense core. Optimizing the beam temperature for the imploded fuel configuration shown in Fig. 1(b), we find an optimal value of $T_{C}$ $=200 \mathrm{MeV}$, for which $\eta_{c}=52 \%$ and $E_{i g}=17.5 \mathrm{~kJ}$, which is again similar to that found for Maxwellian protons.

\section{B. Ignition energies for the super-Gaussian fuel configuration}

Ignition energies as a function of proton temperature and kinetic energy of carbon ions are shown in Figs. 6 and 7, respectively. They have been obtained as the minimum beam energy for which the thermonuclear fusion power has an exponential or higher growth sustained in 


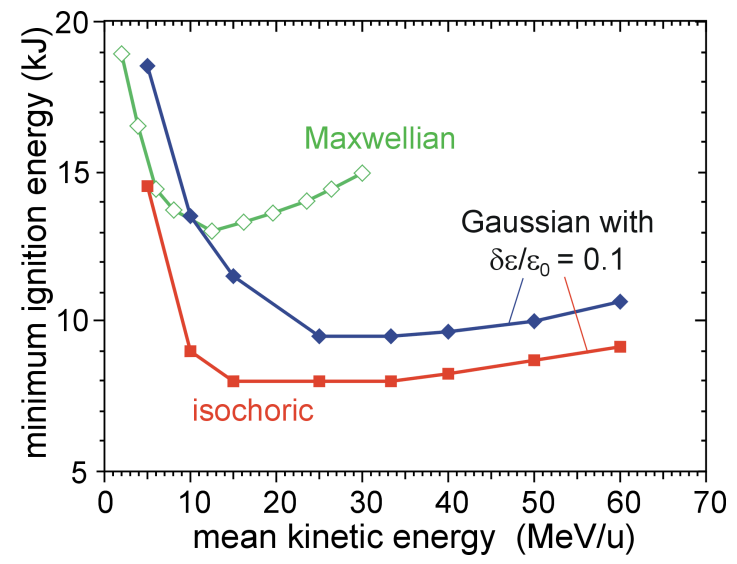

FIG. 7: Minimum ignition energies of the target shown in Fig. 1(a) heated by carbon ions with Gaussian and Maxwellian energy distributions as a function of the mean kinetic energy per nucleon. The ignition energies corresponding to a Gaussian beam in an ideal isochoric fuel are also shown. The ion source - core distances are $d=1.35 \mathrm{~cm}$ for the beams with Gaussian energy distributions and $d=0.5$ $\mathrm{mm}$ for the Maxwellian one.

time. Both figures show that ignition energies increase for low and high ion kinetic energies, reaching a minimum for intermediate values. This is closely related with the pulses used in the simulations. For low ion kinetic energies $\epsilon_{0}$, the pulse has a relatively low power, $P_{\max } \propto \epsilon_{0}^{1 / 2}$, and long duration, $t_{\text {pulse }} \propto \epsilon_{0}^{-1 / 2}$, as obtained from Eq. (1). In this case, the pulse parameters depart from the optimal values and ignition energies increase. For high ion kinetic energies, $E_{i g}$ increases again due to the higher fuel mass heated by the ions. We found an optimal proton temperature $T_{p}$ around $4 \mathrm{MeV}$, for which $E_{i g}=12.7$ $\mathrm{kJ}$. This energy is higher than the $\approx 9 \mathrm{~kJ}$ found in Ref. [9] for an ideal isochoric fuel configuration with a DT density of $500 \mathrm{~g} / \mathrm{cm}^{3}$ and the same source - core distance $d=0.5$ $\mathrm{mm}$. This difference accounts for the energy deposited in the plasma surrounding the dense fuel. For the same ideal isochoric configuration used in the reference, our simulations give $E_{i g}=9.5 \mathrm{~kJ}$, in good agreement with [9]. This result evidences the importance of the plasma surrounding the dense fuel, that increases substantially ignition energies. Thus, the areal density of this plasma should be minimized by the appropriate design of the fuel capsule or by other means, such as the two-beam scheme discussed in Refs. [8, 9, 38].

Quasimonoenergetic carbon ions $\left(\delta \epsilon / \epsilon_{0}=0.1\right)$ have a better coupling with the dense fuel. Figure 7 shows that ignition energies are around $9.5 \mathrm{~kJ}$ for the optimal energy range from 25 to $40 \mathrm{MeV} / \mathrm{u}$, which are about $25 \%$ lower than those obtained for Maxwellian protons (see Fig. 6). Similarly to PFI, the ignition energies can be reduced further by removing the coronal plasma surrounding the dense core. For "ideal" isochoric targets, $E_{i g}$ is reduced to $8 \mathrm{~kJ}$ for $15-32 \mathrm{MeV} / \mathrm{u}$ ions. Note that the effect of the surrounding plasma on $E_{i g}$ is more pronounced for

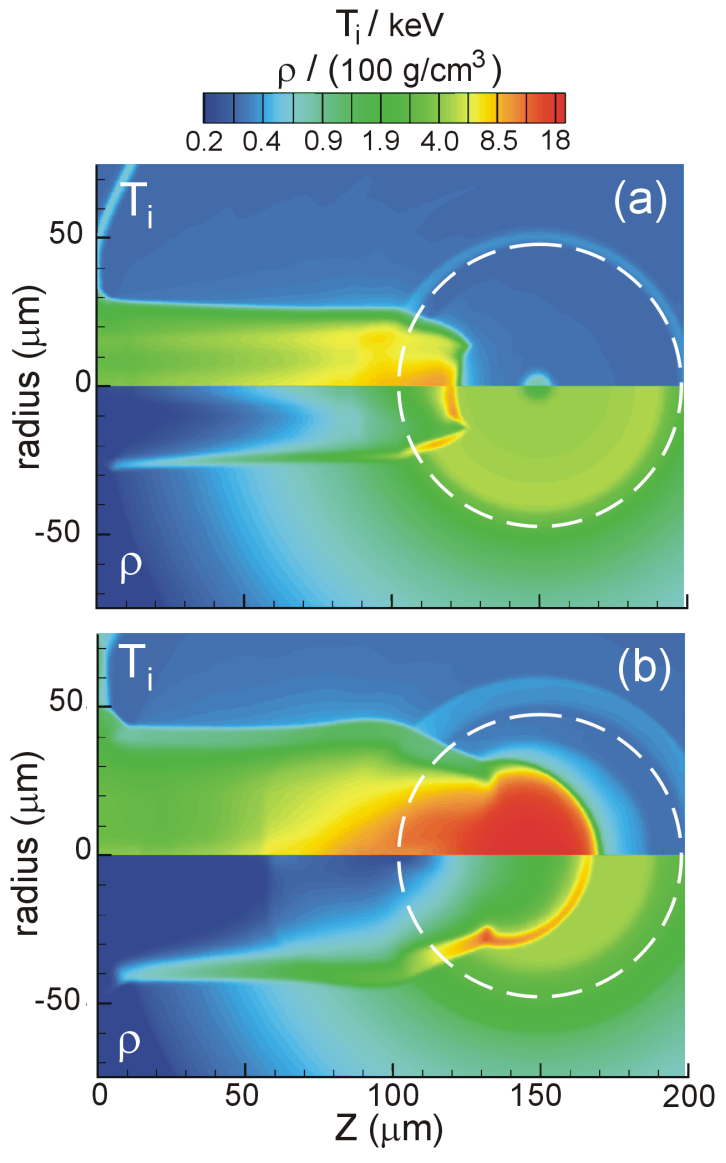

FIG. 8: Ion temperature $\left(T_{i}\right)$ and density $(\rho)$ maps of the target shown in Fig. 1(b) just after the end of the pulse (23 ps) and (b) when the burn wave is propagating (50 ps). The carbon ions have a mean energy of $400 \mathrm{MeV}$ with $\delta \epsilon / \epsilon_{0}=$ 0.1 . The total pulse energy is $12 \mathrm{~kJ}$. Dashed circles show the initial position of the $\rho=200 \mathrm{~g} / \mathrm{cm}^{3}$ isocontour.

ion energies lower than $25 \mathrm{MeV} / \mathrm{u}$.

Maxwellian carbon ions have ignition energies much higher than quasimonoenergetic ions and comparable to those found for Maxwellian protons. For instance, Maxwellian protons and carbon ions have ignition energies around $13 \mathrm{~kJ}$ for the optimal temperatures $T_{p}=4$ $\mathrm{MeV}$ and $T_{C}=100 \mathrm{MeV}$, in agreement with the coupling efficiencies discussed in Sec. II.A.

\section{Ignition energies for the imploded fuel configuration}

We consider in this section quasimonoenergetic carbon ions with different mean kinetic energies $\epsilon_{0}$ and energy spreads $\delta \epsilon / \epsilon_{0}$ heating the imploded fuel configuration shown in Fig. 1(b). Typical fuel density and ion temperature maps at the end of the pulse and during the burn wave propagation are shown in Fig. 8 Ion temperatures around $10 \mathrm{keV}$ in a hot spot of approximately 20 $\mu \mathrm{m}$ diameter can be observed in Fig. 8(a). 


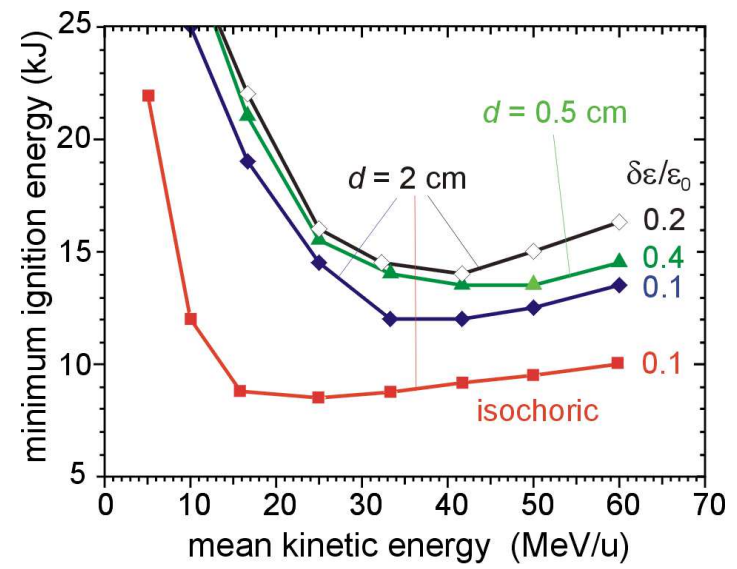

FIG. 9: Minimum ignition energies of the target shown in Fig. 1(b) heated by carbon ion beams with Gaussian energy distributions and different energy spreads $\delta \epsilon / \epsilon_{0}$. The ignition energies corresponding to an ideal isochoric fuel are also shown. The curves labeled by 0.1 and 0.2 have been obtained for an ion source-target distance $d=2 \mathrm{~cm}$, and the curve labeled by 0.4 for $d=0.5 \mathrm{~cm}$.

The ignition energies are shown in Fig. 9] It is remarkable that these energies are at least $25 \%$ higher than those obtained for the target with the super-Gaussian density distribution (see Fig. 7). This is mainly due to the energy deposition in the plasma surrounding the core, as can be evidenced by comparing the ignition energies with those obtained for the ideal isochoric configuration. Note that the effect of the surrounding plasma on $E_{i g}$ is particularly important for relatively low ion kinetic energies. Thus, the optimal energy range of quasimonoenergetic carbon ions is shifted from the $25-40 \mathrm{MeV} / \mathrm{u}$ obtained for the super-Gaussian density distribution to the $33-$ $50 \mathrm{MeV} / \mathrm{u}$ shown by the curve labeled with $\delta \epsilon / \epsilon_{0}=0.1$ in Fig. 9,

The effect of the ion source - core distance $d$ on the ignition energies can be observed by comparing the curves labeled by 'isochoric' in Figs. 7 and 9, The lowest ignition energy changes only slightly, from $8 \mathrm{~kJ}$ in Fig. 7 to $8.5 \mathrm{~kJ}$ in Fig. 9, when the distance $d$ increases from 1.35 $\mathrm{cm}$ to $2 \mathrm{~cm}$, respectively. Its effect is more pronounced, however, for ions with energies $\epsilon_{0}<10 \mathrm{MeV} / \mathrm{u}$.

Ignition energies are also sensitive to the ion beam energy spread, as can be seen by comparing the curves for $\delta \epsilon / \epsilon_{0}=0.1$ and 0.2 in Fig. 9. Spreads higher than 0.2 lead to very high ignition energies. For instance, the ignition energies for $\delta \epsilon / \epsilon_{0}=0.4$ and a distance $d=2 \mathrm{~cm}$ become higher than $20 \mathrm{~kJ}$. This energy can be reduced by placing the ion source closer to the target. Thus, the ignition energy of $50 \mathrm{MeV} / \mathrm{u}$ carbon ions with $\delta \epsilon / \epsilon_{0}=0.4$ generated at a distance $d=0.5 \mathrm{~cm}$ turns out to be $E_{i g}$ $=13.5 \mathrm{~kJ}$, slightly higher than the $12.5 \mathrm{~kJ}$ obtained for ions with the same kinetic energy, $\delta \epsilon / \epsilon_{0}=0.1$ and $d=2$ $\mathrm{cm}$. Yet, a source - core distance $d=0.5 \mathrm{~cm}$ is still high enough to use target designs without a re-entrant cone inserted. Therefore, we can conclude that the requirement

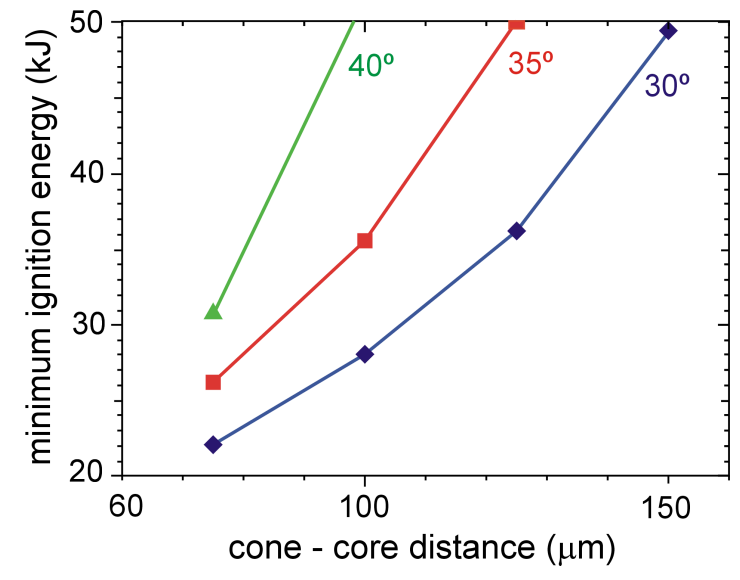

FIG. 10: Minimum ignition energies of an electron beam with mean kinetic energy of $1.6 \mathrm{MeV}$ impinging on the target shown in Fig. 1(a). Cone - core distance is the distance between the left surface of the simulation box and the center of the super-Gaussian density distribution. The curves are labeled with the initial divergence half-angle of the relativistic electron beam.

of quasimonoenergetic spectra can be relaxed in such a manner that ion beams with broad energy distributions generated relatively far from the compressed core can ignite realistic imploded fuel configurations with moderate beam energies. If the plasma surrounding the dense core is minimized or removed, these ignition energies could be reduced further.

\section{COMPARISON WITH RELATIVISTIC ELECTRON BEAMS}

Recent results on electron-driven fast ignition with a fuel configuration similar to that depicted in Fig. 1(a) have shown that ignition energies depends strongly on beam divergence and distance from the cone-tip to the dense fuel $(150 \mu \mathrm{m}$ in Fig. [1(a)) 39]. We found that beam focusing by self-generated magnetic fields plays an important role, reducing substantially the ignition threshold. The ignition energies for $1.6 \mathrm{MeV}$ electrons are plotted in Fig. 10, Targets with cone - core distances lower than $125 \mu \mathrm{m}$ can be ignited by electron beams with energies around $40 \mathrm{~kJ}$ if the beam divergence half-angle at the cone tip is lower than $30-35^{\circ}$. Assuming a laser-to-fast electron conversion efficiency of $40 \%$, this energy corresponds to an energy of the multi-petawatt laser system around $100 \mathrm{~kJ}$.

Our results on ion fast ignition show that the target pictured in Fig. 1(a) can be ignited by a Maxwellian proton beam of $12.7 \mathrm{~kJ}$ with the optimal temperature $T_{p}=$ $4 \mathrm{MeV}$. This energy falls to $9.5 \mathrm{~kJ}$ for quasimonoenergetic carbon ions in the energy range of $25-40 \mathrm{MeV} / \mathrm{u}$. Assuming a laser-to-ion conversion efficiency of $10 \%$, we find that fast ignition driven by quasimonoenergetic ions requires laser energies around $100 \mathrm{~kJ}$, similar to those 
found for fast ignition by relativistic electrons. Thus, the better coupling with the plasma, the lower energy requirements and the possibility to use of targets without cones set quasimonoenergetic ions as a promising candidate to demonstrate fast ignition if conversion efficiencies around $10 \%$ can be achieved experimentally (by means of either the laser breakout afterburner or radiation pressure acceleration schemes).

Our results also show that the ion beam energy requirements for more realistic imploded target configurations, such as that shown in Fig. 1(b), are around 25\% higher (see Fig. 9). In this case, the flexibility offered by iondriven fast ignition can be used to further reduce the ignition energies. The use of multiple ion beams [9, 38] or ions heavier than carbon may be an option to reduce the laser energy requirements. On the other hand, if the ion energy spread is higher than the $10 \%$ assumed here as reference, our calculations show that there is still margin to reduce the ion source - core distance maintaining target designs without a re-entrant cone inserted.

\section{CONCLUSIONS}

Fast ignition driven by ions presents several advantages over fast ignition driven by relativistic electrons such as their well know interaction with the plasma, their focusability and the flexibility for the fuel ignition, such as the possibility of using multiple beams. This last possibility is particularly useful to minimize the areal density of the plasma surrounding the dense core and therefore to reduce significantly the ignition energies. The use of quasimonoenergetic ions heavier than protons improves the beam coupling efficiency and reduces substantially the ignition energies. In addition, quasimonoenergetic ions allow to place the ion source far away from the fuel capsule, simplifying target design and fuel implosion and compression, provided that ions can be focused onto a spot of about $30 \mu \mathrm{m}$ over distances of a few centimetres. This conclusion is still valid for ion energy spreads as high as $40 \%$. In this case, the increase of the ignition energy can be compensated by reducing the source - capsule distance, which is still high enough to avoid the use of re-entrant cones.

Prior to the application of the fast ignition by quasimonoenergetic ions scheme, laser to ion conversion efficiencies of the order of $10 \%$ have to be demonstrated experimentally. If these conversion efficiencies are achieved, our calculations show that the laser energy requirements are similar to those found for fast ignition driven by relativistic electrons. In this case, the advantages of the ion fast ignition scheme are attractive enough to envision ion fast ignition as a candidate to demonstrate fast ignition in future facilities such as HiPER [10]

Future studies will include advanced beam configurations, realistic beam divergences and the consideration of other ions.

\section{Acknowledgments}

One of the authors (JJH) would like to thank the fruitful discussions and the hospitality of the P-24 group of LANL. MT has been supported by a contract of the Spanish Ministry of Education (Ramón y Cajal 2007-0447). This work was partially supported by the research grants ENE2006-06339 and CAC-2007-013 of the Spanish Ministry of Education and by the IFE Keep-in-touch Activities of EURATOM.
[1] M. Roth, T.E. Cowan, M.H. Key, S.P. Hatchett, C. Brown, W. Fountain, J. Johnson, D.M. Pennington, R.A. Snavely, S.C. Wilks, K. Yasuike, H. Ruhl, F. Pegoraro, S.V. Bulanov, E.M. Campbell, M.D. Perry and H. Powell, Phys. Rev. Lett. 86, 436 (2001).

[2] M. Roth, Plasma Phys. Control. Fus. 51, 014004 (2009).

[3] M. Tabak. J. Hammer, M.E. Glinsky, W.L. Kruer, S.C. Wilks, J. Woodworth, E.M. Campbell, M.D. Perry and R.J. Mason, Phys. Plasmas 1, 1626 (1994).

[4] R.A. Snavely, M.H. Key, S.P. Hatchett, T.E. Cowan, M. Roth, T.W. Phillips, M.A. Stoyer, E.A. Henry, T.C. Sangster, M.S. Singh, S.C. Wilks, A. MacKinnon, A. Offenberger, D.M. Pennington, K. Yasuike, A.B. Langdon, B.F. Lasinski, J. Johnson, M.D. Perry, and E.M. Campbell, Phys. Rev. Lett. 85, 2945 (2000).

[5] L. Robson, P.T. Simpson, R.J. Clarke, K.W.D. Ledingham, F. Lindau, O. Lundh, T. McCanny, P. Mora, D. Neely, C.-G. Wahlstrm, M. Zepf and P. McKenna, Nat. Phys. 3, 58 (2007).

[6] K.A. Flippo, E. d'Humières, S.A. Gaillard, J. Rassuchine, D.C. Gautier, M. Schollmeier, F. Nürnberg, J.L. Kline, J. Adams, B. Albright, M. Bakeman, K. Harres, R.P.
Johnson, G. Korgan, S. Letzring, S. Malekos, N. RenardLeGalloudec, Y. Sentoku, T. Shimada, M. Roth, T. E. Cowan, J. C. Fernndez,1 and B. M. Hegelich, Phys. Plasmas 15, 056709 (2008).

[7] J. Fuchs, P. Antici, E. d'Humières, E. Lefebvre, M. Borghesi, E. Brambrink, C. A. Cecchetti, M. Kaluza, V. Malka, M. Manclossi, S. Meyroneinc, P. Mora1, J. Schreiber, T. Toncian, H. Pèpin and P. Audebert, Nat. Phys. 2, 48 (2006).

[8] M. Temporal, Phys. Plasmas 13, 122704 (2006).

[9] M. Temporal, J.J. Honrubia and S. Atzeni, Phys. Plasmas 15, 052702 (2008).

[10] M. Dunne, Nature Physics 2, 2 (2006).

[11] R. Ramis and J. Ramirez, Nucl. Fus. 44, 720 (2004).

[12] A. Macchi, F. Cattani, T.V. Liseykina and F. Cornolti, Phys. Rev. Lett. 94, 165003 (2005).

[13] A.P.L. Robinson, M. Zepf, S. Kar, R.G. Evans and C. Bellei, New J. Phys. 10, 013021 (2008).

[14] O. Klimo, J. Psikal, J. Limpouch and V. Tikhonchuk, Phys. Rev. ST Accel. Beams 11, 031301 (2008).

[15] S.G. Rykovanov, J. Schreiber, J. Meyer-ter-Vehn, C. Bellei, A. Heing, H.C. Wu and G. Geissler, New J. Phys. 
10, 113005 (2008).

[16] N. Naumova, T. Schlegel, V.T. Tikhonchuk, C. Labaune, I.V. Sokolov and G. Mourou, Phys. Rev. Lett. 102, 025002 (2009).

[17] B. Qiao, M. Zepf, M- Borghesi and M. Geissler, Phys. Rev. Lett. 102, 145002 (2009).

[18] M. Chen, A. Pukhov, T.P. Yu and Z.M. Sheng, Phys.Rev. Lett. 103, 024801 (2009).

[19] L. Yin, B.J. Albright, B.M. Hegelich, K.J. Browers, K.A. Flippo, T.J.T. Kwan and J.C. Fernández, Phys. Plasmas 14, 056706 (2007).

[20] J.C. Fernández, B.J. Albright, K.A. Flippo, B.M. Hegelich, T.J. Kwan, M.J. Schmitt and L. Yin, J. Phys.: Conf. Ser. 112, 022051 (2008).

[21] J.C. Fernández, J.J. Honrubia, B.J. Albright, K.A. Flippo, D.C. Gautier, B.M. Hegelich, M.J. Schmitt, M. Temporal and L. Yin, Nucl. Fus. 49, 065004 (2009).

[22] B.J. Albright, M.J. Schmitt, J.C. Fernández, G.E. Cragg, I. Tregillis, L. Yin and B.M. Hegelich, J. Phys.: Conf. Ser. 112, 022029 (2008).

[23] M.L. Shmatov, J. Phys.: Conf. Ser. 112, 022061 (2008).

[24] J. Badziak, Opto-Electron. Rev. 15, 1 (2007).

[25] S. Atzeni, A. Schiavi, J.J. Honrubia, X. Ribeyre, G. Schurtz, Ph. Nicolai, M. Olazabal-Loumé, C. Bellei, R.G. Evans and J.R. Davies, Phys. Plasmas 15, 056311 (2008).

[26] M. Dunne, N. Alexander, F. Amiranoff, P. Auger, S. Atzeni, H. Azechi, V. Bagnoud, P. Balcou, J. Badziak, D. Batani, C. Bellei, D. Besnard, R. Bingham, J. Breil, M. Borghesi, S. Borneis, A. Caruso, J. C. Chanteloup, R. J. Clarke, J. L. Collier, J. R. Davies, J.-P. Dufour, P. Estrailler, R. L. Evans, M. Fajardo, R. Fedosejevs, G. Figueria, J. Fils, J. L. Feugeas, M. Galimberti, J.-C. Gauthier, A. Giulietti, L. A. Gizzi, D. Goodin, G. Gregori, S. Guskov, L. Hallo, C. Hermandez-Gomez, D. Hoffman, J. Honrubia, S. Jacquemot, M. Key, J. Kilkenny, R. Kingham, M. Koenig, F. Kovacs, A. McEvoy, P. McKenna, J. T. Mendonca, J. Meyer-ter-Vehn, K. Mima, G. Morou, S. Moustazis, Z. Najmudin, P. Nickles, D. Neely, P. Norreys, M. Olazabal, A. Offenberger, N. Papodogianis, J. M. Perlado, J. Ramirez, R. Ramis, Y. Rhee, X. Ribeyre, A. Robinson, K. Rohlena, S. Rose, M. Roth, C. Rouyer, C. Rulliere, B. Rus, W. Sandner, A. Schiavi, G. Schurtz, A. Sergeev, M. Sherlock, L. Silva, R. Smith, G. Sorasio, C. Strangio, H. Takabe, M. Tatarakis, V. Tikhonchuk, M.
Tolley, M. Vaselli, P. Velarde, T. Winstone, K. Witte, J. Wolowski, N. Woolsey, B. Wyborn, M. Zepf, and J. Zhang, HiPERTechnical Background and Conceptual Design Report 2007, Rutherford Appleton Laboratory, Report No. RAL- TR-2007-008 (June 2007), available on the site www.hiper-laser.org.

[27] H. Azechi, T. Jitsuno, T. Kanabe, M. Katayama, K. Mima, N. Miyanaga, M. Nakai, S. Nakai, H. Nakaishi, M. Nakatsuka, A. Nishiguchi, P.A. Norreys, Y. Setsuhara, M. Takagi, M. Yamanaka and C. Yamanaka, Las. Part. Beams 9, 193 (1991).

[28] J.J. Honrubia and J. Meyer-ter-Vehn, J. Phys.: Conf. Ser. 112, 022055 (2008).

[29] D.S. Clark and M. Tabak, Nucl. Fusion 47, 1147 (2007).

[30] J.J. Honrubia, J. Quant. Spectrosc. Radiat. Transfer 49, 491 (1993).

[31] J.J. Honrubia, Charged particle transport in Fusion by Inertial Confinement, G. Velarde, Y. Ronen and J.M. Martínez-Val (Eds.), CRC Press, Boca Ratón, Florida (1993).

[32] S. Atzeni, M. Temporal and J.J. Honrubia. Nucl. Fus. 42, L1 (2002).

[33] M. Temporal, J.J. Honrubia and S. Atzeni, Phys. Plasmas 9, 3098 (2002).

[34] P.K. Patel, A.J. Mackinnon, M.H. Key, T.E. Cowan, M.E. Foord, M. Allen, D.F. Price, H. Ruhl, P.T. Springer and R. Stephens, Phys. Rev. Lett. 91, 125004 (2003).

[35] M.H. Key, Phys. Plasmas 14, 055502 (2007).

[36] T. Toncian, M. Borghesi, J. Fuchs, E. d'Humières, P. Antici, P. Audebert, E. Brambrink, C.A. Cecchetti, A. Pipahl, L. Romagnani and O. Willi, Science 312, 410 (2006).

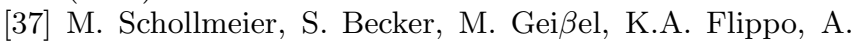
Blazevic, S.A. Gaillard, D.C. Gautier, F. Gruner, K. Harres, M. Kimmel, F. Nurnberg, P. Rambo, U. Schramm, J. Schreiber, J. Schutrumpf, J. Schwarz, N.A. Tahir, B. Atherton, D. Habs, B.M. Hegelich, and M. Roth, Phys. Rev. Lett. 101, 055004 (2008).

[38] M. Temporal, R. Ramis, J.J. Honrubia and S. Atzeni, Plasma Phys. Control. Fus. 51, 035010 (2009).

[39] J.J. Honrubia and J. Meyer-ter-Vehn, Plasma Phys. Control. Fus. 51, 014008 (2009). 